\title{
Induced polar materials for intense radiation monitoring
}

\author{
I.V. Blonsky, V.F. Kosorotov, L.V. Shchedrina and L.V. Levash \\ Institute of Physics National Academy of Sciences of Ukraine \\ 46, prospect Nauki, 03650, Kiev, Ukraine \\ I.V.Blonsky: E-mail: blon@iop.kiev.ua.Phone:(044)265 3138, Fax: (044) 2651543 \\ V.F. Kosorotov: E-mail: shched@iop.kiev.ua.Phone: (044) 2656269 \\ L.V. Shchedrina:E-mail: shched@phys.semicond.kiev.ua.Phone: (044) 2656269 \\ L.V. Levash: E-mail:levash@iop.kiev.ua.Phone: (044) 2657942
}

\begin{abstract}
Theoretical and experimental researches on the electric potential spatial distribution in thin crystal plates of zinc selenide and quartz, presented in this paper, is a constituent part of a common approach elaborated for a study of the polar states induced by inhomogeneous heating in a wide class of materials including those not belonging to the polar crystallographic classes. Construction original principles of a new class of the transmission-type power pyroelectric sensors with a maximum attainable upper limit of the dynamic range for an intense radiation monitoring in a wide region of IR spectrum are developed.
\end{abstract}

Keywords: pyroelectricity, spatially inhomogeneous heating, induced polar state, transmission-type power sensors

Paper received 05.10.99; revised manuscript received 03.11.99; accepted for publication 14.03.00.

\section{Introduction}

Well-known semiconductor and dielectric materials belonging to the ten polar classes are widely used as pyroactive matters in the pyroelectric devices for infrared detection and thermal imaging [1]. Traditional pyroelectric IR sensors based on the primary and secondary pyroelectric effects turned out to have low efficiency for measurements of high-power laser radiation due to a strong absorption of radiation fluxes, which causes a destruction of a sensor sensitive element. The potentialities of available at present devices, including the splitting or deflection of some part of a laser beam, have been exhausted [2]. As a consequence of a strong temperature dependence of electric characteristics of ferroelectric materials in the vicinity of the phase transition temperature $T_{\mathrm{c}}$ the measurement of radiation parameters over the temperature region near $T_{\mathrm{c}}$ proves to be impossible owing to severe nonlinear distortions of a signal.

To overcome the outlined difficulties encountered under measuring intensive laser radiation, we, for the first time, advanced using nonpolar piezoelectrics that do not belong to pyroelectric classes and therefore exhibit high transparency in a particular spectral region, for a power radiation monitoring [3]. These crystals reveal pyroactive properties under nonequilibrium thermal conditions only. It has been shown $[4,5]$ that the presence of the temperature gradient causing the nonuniformly stressed state of the crystal gives rise to a polarization which is piezoelectric in nature and is determined by a character of thermoelastic stresses. Tertiary pyroelectric effect (TPE) due to this polarization can arise in all twenty noncentral crystallographic classes, which essentially extends the class of crystals possessing the required optical, electric and thermoelastic properties. These can be used as sensitive elements of IR sensors.

Previously the pyroelectric effect has been investigated under nonequilibrium thermal conditions in crystals of the ten polar classes [6-10]. The rapid research on polarization phenomena under inhomogeneous heating in recent years is conducted in nonpolar piezoelectrics [11-14]. In spite of the fact that TPE shares a common nature with the secondary pyroelectric effect, it differs fundamentally from both the primary and secondary pyroelectric effects by its symmetry resulting in TPE existence in the crystals not belonging to the polar classes. It has been just this TPE property which has led to the development of IR sensors of a new generation. Theoretical and experimental explorations are aimed at the investigation of physical nature of induced polar states and mechanisms of their 


\section{I.V. Blonsky et al.: Induced polar materials for intense radiation monitoring}

appearance in nonpolar piezoelectrics. New approach to the development of infrared sensors and devices on the base of the polarization phenomena under investigation is formulated.

\section{Polar states in thin crystal plates}

Let us consider a thin crystal plate subjected to axisymmetric heating by a radiation sinemodulated flux with a wavelength in the crystal transparency band, i.e. when the temperature in a round plate changes only along its radius. Sensors based on the sensitive elements with indicated temperature distribution known to be the transmission-type power sensors. The complete system of equations describing the thermodynamic state of a crystal local volume in the field of thermal, mechanical and electric perturbations has been described in our study [15]. The analysis of the polarization spatial distribution in it presents considerable mathematical difficulties, and hence we shall proceed from the following approximation: a plane-stressed state will be realized in a thin plate, being an arbitrary cut of the crystal, if an average (over the plate thickness) tensor of thermoelastic stresses $\sigma_{i k}$ is introduced. Those tensor components, which are equal to zero on mechanically free front surface of the plate, are supposed to be zero in its volume, too. Then, averaged equilibrium equations are automatically satisfied by entering the stress function $F(x, y)$ :

$$
\sigma_{x x}=\partial^{2} F / \partial y^{2}, \sigma_{y y}=\partial^{2} F / \partial x^{2}, \sigma_{x y}=-\partial^{2} F / \partial x \partial y \text {. }
$$

In a general case the equation for function $F$ is as follows:

$$
\begin{aligned}
& S_{22} \frac{\partial^{4} F}{\partial x^{4}}+S_{11} \frac{\partial^{4} F}{\partial y^{4}}+\left(2 S_{12}+S_{66}\right) \frac{\partial^{4} F}{\partial x^{2} \partial y^{2}}- \\
& -2 S_{16} \frac{\partial^{4} F}{\partial x \partial y^{3}}-2 S_{26} \frac{\partial^{4} F}{\partial x^{3} \partial y}= \\
& =\alpha_{6} \frac{\partial^{2} \theta}{\partial x \partial y}-\alpha_{1} \frac{\partial^{2} \theta}{\partial y^{2}}-\alpha_{2} \frac{\partial^{2} \theta}{\partial x^{2}}
\end{aligned}
$$

were $S_{\lambda v}, a_{\lambda}$ are the tensor components of elastic compliance and heat expansion, $\theta$ is increase in the crystal temperature. All tensor magnitudes must be taken in the coordinate system concerned with a plate.

Comprehensive study of the polarization spatial distribution in a general case is unsolvable problem. Equation (2) can be solved only in exceptional cases. It has been possible to carry out investigation of TPE for the crystals, belonging to the classes $D_{3}, C_{3 \mathrm{v}}, T_{d}$ and some classes of hexagonal syngony having a triad rotation axis $\left(D_{6}, C_{6}, C_{6 \mathrm{v}}, D_{3 \mathrm{~h}}, C_{3 \mathrm{~h}}\right)$. Let us discuss the results obtained in more details for crystals of classes $T_{d}$ and $D_{3}$. This choice is not random. As it will be seen from the follow- ing section, analysis of optical and electromechanical properties of these crystals revealed good potentialities for their use as sensitive elements of sensors based on TPE. We were able to develop a new class of transmission-type power pyroelectric sensors possessing the maximum attainable high upper limit of the dynamic range for a middle infrared spectral region on the basis of the single crystals of zinc selenide and for the visible and near infrared ranges on the basis of the quartz crystal.

The possibility of a practical utilization of the polar states induced by inhomogeneous heating becomes available only if the spatial distribution of the electric potential in the crystal is found. In regard to $\mathrm{ZnSe}$, oriented crystals (normal to the plate is directed along the direction [111] coincident with a three-fold symmetry axis) were studied. As the symmetry analysis indicates, in these cuts longitudinal (induced polarization vector is directed along incident radiation flux normally to the plate plane) and transverse (the polarization vector perpendicular to the radiation flux lies in the plate plane) effects exist simultaneously in the region remained cold (with respect to the temperature variable component). A longitudinal component of the electric field caused by the polarization $P_{\mathrm{Z}}$ determines the longitudinal TPE. The distribution of a bound charge density on the surface perpendicular to the disk axis has the form:

$$
P_{z}=P_{z}^{\prime}\left[\theta(r)-\frac{2 V\left(R_{0}\right)}{R_{0}^{2}}+\frac{6 b G\left(R_{0}\right)}{R_{0}}\left(1-\frac{2 r^{2}}{R_{0}^{2}}\right)\right]
$$

where

$$
\begin{aligned}
& G(r)=V(r) / r-\left(4 / r^{3}\right) \int_{0}^{r} V\left(r^{\prime}\right) r^{\prime} d r^{\prime}, \\
& V(r)=\int_{0}^{r} \theta\left(r^{\prime}\right) r^{\prime} d r^{\prime},
\end{aligned}
$$

$R_{0}$ is the disk radius, $b$ is some coefficient (rather cumbersome) allowed for a crystal anisotropy. This distribution is characterized by the change of a charge sign in the heated and non-heated regions. The total bound charge released on any its surface is zero, and this distinguishes the effect in question from the primary and secondary effects, where charge compensation is possible only when the charges are summed over the whole surface of the disk. Signal electrodes in the form of rings are deposited on the disk front surfaces and cover only its non-heated region.

There is a transverse component of the electric field in the plate plane associated with a bound charge on the lateral surface of the plate

$$
P_{r}=P_{r}^{\prime}\left[\theta(r)-\frac{2 V(r)}{r^{2}}+\frac{6 b G\left(R_{0}\right) r^{2}}{R_{0}^{3}}\right] \sin 3 \alpha .
$$




\section{I.V. Blonsky et al.: Induced polar materials for intense radiation monitoring}

As evident from (4), the configuration of the electrode system is totally dependent on the symmetry of the transverse TPE, during which an angular distribution of the potential on the lateral surface is determined by $\sin 3 \alpha$. A system of electrically insulated from one another electrodes has to be deposited on unipolar parts of the lateral surface, in which the potentials should be of the same sign. The electrodes covering sections with charges identical in sign are connected into one end, and the remaining electrodes - another one. Both leads are connected to the standard measuring circuit. The potential nonzero difference of which appearing of these electrodes can provide information on the incident radiation flux.

Let us consider the polar states in quartz crystal. Equation (2) for the $Z$-cut is solved exactly, and the stress function is such that components $\sigma_{i k}$ calculated from (1) are equal to expressions, obtained on the basis of isotropic model (with a precision of constant). The polarization nonzero components in the polar coordinate system are as follows

$$
\begin{aligned}
& P_{r}=P_{r}^{\prime}\left[\theta(r)-\frac{2 V(r)}{r^{2}}\right] \cos 3 \alpha, \\
& P_{\varphi}=-P_{\varphi}^{\prime}\left[\theta(r)-\frac{2 V(r)}{r^{2}}\right] \sin 3 \alpha .
\end{aligned}
$$

Calculating a bound charge in the plate and solving electrodynamic part of the problem like that in [16], the expression for the electric potential can be obtained. An important point is that only the transverse TPE takes place in the $Z$-cut quartz, and its angle dependence is determined by $\cos 3 \alpha$. If it is granted that irradiation region is subjected to homogeneous heating, the polar state is lacking in it. Polarization arises abruptly on the boundary of heated and cold zones. Such a polarization spatial distribution is supported by the symmetry analysis performed in [15]. Experimental results on the potential angle distribution correlate well with the theoretical ones (5). Electrode system is formed in the same manner as for ZnSe crystal.

It is necessary to make a few remarks with reference to the $X_{1}$ and $X_{2}$-cuts. Equation (2) has long resisted solution for these cases and consequently approximate methods should be employed. The simplest of all is the approach when the problem elastic part is assumed as isotropic, whereas electrodynamic part is solved exactly. More exact approximation is consideration of heated and cold regions of the plate independently of one another. The polarization distribution in the heated region is derived from a complete system of equations approximately allowing for an absolute restriction of deformations in the plate plane. For a cold region a purely mechanical problem with definition of the thermoelastic tensor on its internal surface and on free from forces external one should be solved. It should be noted that approximations indicated lead to different radial dependences of the electric potential and to the same angle one.
The overall picture of the potential distribution may be established on the basis of the symmetry approach elaborated in [15]. Polar state in a heated zone of the $X_{2^{-}}$ cut quartz is determined by the symmetry group $G=2$ with a symmetry axis in the cut plane. Polar state in a cold region exists under dissymmetry conditions. Both regions of the plate make a contribution to the potential on the plate lateral surface, however, calculations point to the fact that the potential distribution is invariant under the twofold symmetry axis.

The $X_{1}$-cut of quartz is characterized by following features. Polar state in both heated and cold regions is determined by the symmetry group $G=2$ with a symmetry axis directed normally to the cut plane. Therefore, only the longitudinal TPE is exhibited in both regions. Signal electrodes for a given case are the system consisting of two congruent electrodes in a form of rings deposited on the plate opposite faces. Internal diameter of rings is larger than diameter of a laser beam.

\section{Optimization of the crystal physical proper- ties}

The investigation commencement of the polar states induced by inhomogeneous heating have been aimed at searching new effective methods for a measurement of radiation intense fluxes of technological lasers. In this connection the essential feature required of a pyroelectric sensor acting on TPE is that there should be a high upper limit of the dynamic range $W$. For the sensors under consideration high values for $W$ are achieved in the band of the crystal maximal transparency. Therefore, the value of the absorption coefficient $\mu$ (in $\left.\mathrm{cm}^{-1}\right)$ at the wave length of a measurable radiation is the principal characteristic of the crystal. Another feature (no less important) determining the value of $W$ is the crystal radiation resistance. If it is granted that the material radiation resistance is qualitatively characterized by some temperature limiting increase $\theta_{\text {lim }}$, the following expression for $W$ can be approximately obtained

$W=\left(\theta_{\lim } \cdot c \rho\right) /\left(\mu \cdot \tau_{T}\right)$,

where $\tau_{T}$ is the time thermal constant of the sensor, $c \rho$ is the specific heat per unit volume. The criterion (6) is derived from the condition - the equilibrium value of the temperature increase must be equal to the temperature limiting increase. A magnitude of $\theta_{\text {lim }}$ can be determined by both the crystal destruction temperature (this is an ideal case) and temperature under which processes destroying the polar state in the crystal are feasible (phase transitions or twinning processes).

Brief mention should be made of the role of the measurement thermal conditions. As may be inferred from the relationship (6), the thermal conditions are essential to the measurement of radiation intense continuous fluxes. Firstly, intensity of a heat exchange of the sensor sensitive element with environment determines a value of the 


\section{I.V. Blonsky et al.: Induced polar materials for intense radiation monitoring}

equilibrium temperature in the element. Secondly, in common practice, the upper limit of the dynamic range depends to a large extent on the method of crystal putting under radiation load, as well as the crystal removal from it. In regard to measurements of pulse fluxes, thermal conditions are of no significance, and the value of $\theta_{\lim }$ at a given wavelength generally depends on the measurable radiation parameters (both the energy density in a pulse and a pulse duration).

From the above reasoning it can be said with confidence that the crystals used as windows in a spectrum appropriate region show considerable promise as materials for sensitive elements of transmission-type power pyroelectric sensors. Indicated crystals must not belong to the central crystallographic classes. It is required that they possess small intrinsic conductivity and were free from any temperature anomalies of their physical properties (for instance, permittivity or piezoelectric moduli). Quartz crystals were chosen as sensitive elements for transmission-type power sensors in visible and near infrared spectrum region, and single crystals of zinc selenide for radiation monitoring in the middle IR spectral region ( $\mathrm{CO}_{2}$ laser).

\section{Conclusions}

External vector influence in a form of a spatially inhomogeneous heating of the crystal is proposed as a promising alternative method for a creation of new induced pyroactive structures based on existing nonpolar piezoelectric crystals. These materials are of a great variety on their optical, electric and thermoelastic properties, which largely alleviates metrological problems when measuring intense laser emission. Advanced symmetry analysis of polarization phenomena under nonequilibrium thermal conditions is found to be a highly effective tool for elucidation of physical mechanisms of the induced polar states formation. A new approach to the development of IR pyroelectric sensors as well as devices based on them and used for IR radiation monitoring is elaborated.

\section{References}

1. V.F. Kosorotov, L.S. Kremenchugskij, V.B. Samoilov and L.V.Shchedrina, Pyroelectric Effect and Its Practical Applications, Naukova Dumka, Kiev(1987).

2. R.W. Whatmore, Pyroelectric ceramics and devices for thermal infrared detection and imaging //Ferroelectrics, 118(1/4), pp. 241-259 (1991).

3. V.F. Kosorotov, L.S. Kremenchugskij and L.V. Shchedrina, Tertiary pyroelectric effect and its application for registration of the pulse radiation //Proc. of Conference on Production and Employment of Ferro- and Piezomaterials, Moscow, pp.71-75 (1984).

4. W.Cady, Piezoelectricity, New York, London: McGraw-Hill Book Com., Inc.(1946).

5. J.F. Nye, Physical Properties of Crystals. Oxford University Press, London (1957).

6. I.B. Schein, P.J. Cressman and L.E. Cross, Electrostatic measurement of tertiary pyroelectricity in partially clamped $\mathrm{LiNbO}_{3} / /$ Ferroelectrics, 22(3/4), pp. 945-948 (1979).

7. E.N. Pirogov, B.L. Timan and V.M. Fesenko, Secondary pyroelectric effect in $\mathrm{LiNbO}_{3}$ and $\mathrm{LiTaO}_{3}$ under axisymmetric heating // Crystallography, 27 (6), pp. 1131 -1135(1982).

8. V.F. Kosorotov, L.S. Kremenchugskij, L.V. Levash, L.V. Shchedrina, Tertiary pyroelectric effect in lithium niobate and lithium tantalate crystals//Ferroelectrics, 70(1/2), pp. $27-37$ (1986).

9. V.F. Kosorotov, L.S. Kremenchugskij, L.V. Levash and L.V. Shchedrina, Some specific features in the behavior of dynamic pyroelectric effect under temperature gradient conditions// Ferroelectrics, 118 (1/4), pp. 233 - 240 (1991).

10. X.S. Wang, A Discussion on Type II Pyroelectric Detectors //Infrared Phys. Ferroelectrics Lett. Sec., 15 (5/6), pp. 159-165 (1993).

11. V.F. Kosorotov, L.V. Levash, L.V. Shchedrina et al. Power sensors, based on the tertiary pyroelectric effect, combined with exit windows of CO and $\mathrm{CO}_{2}$ lasers //Quantum Electronics, 24(6), pp. 543-545(1994).

12. T.K. Munshi, K.K. Kundu, R.K.Mahalanabis, Mechanical response in an N-electrode piezo-quartz bar under thermal excitations //Acta Physica Polonica A , 87 (6), pp. 995 - 1002 (1995).

13. I.V. Blonsky, V.F. Kosorotov, , L.V. Shchedrina and L.V. Levash, New pyroactive structures for infrared optoelectronics //SPIE Proc., 3890, pp. 55-62(1999).

14. V.F. Kosorotov, Symmetry properties of tertiary pyroelectric effect in inhomogeneous one-dimensional temperature fields //Inorganic Materials, 31 (6), pp. 827 - 830 (1995) .

15. V.F. Kosorotov, L.V. Shchedrina, L.V.Levash, Creation physical principles of induced pyro-active structures with controllable polar properties //Ukr. J. Phys., 44(1/2), pp.251-258 (1999) .

16. V.F. Kosorotov, A study on electric power detector acting as output windows of $\mathrm{CO}$ and $\mathrm{CO}_{2}$ lasers //SPIE Proc., 2259, pp. 213 - 219 (1994). 